\title{
Hot isostatic pressing of bulk magnesium diboride: mechanical and
}

\section{superconducting properties}

\author{
S.S. Indrakanti, ${ }^{a}$ V.F. Nesterenko, ${ }^{\mathrm{a}, \mathrm{d}^{*}}$ M.B. Maple, ${ }^{\mathrm{b}, \mathrm{c}}$ N.A. Frederick,,${ }^{\mathrm{b}, \mathrm{c}}$ \\ W.M. Yuhasz, ${ }^{\mathrm{c}, \mathrm{d}}$ Shi Lib ${ }^{\mathrm{b}, \mathrm{c}}$ \\ ${ }^{a}$ Department of Mechanical and Aerospace Engineering; ${ }^{b}$ Department of Physics; ${ }^{C}$ Institute for Pure and Applied \\ Physical Sciences; ${ }^{d}$ Materials Science and Engineering Program; University of California, San Diego, La Jolla, CA \\ 92093, USA
}

\begin{abstract}
Two different hot isostatic pressing cycles (HIPing) were investigated to synthesize bulk $\mathrm{MgB}_{2}$ samples: a "standard" cycle where a low vessel pressure is maintained while heating to the process temperature with a subsequent simultaneous pressure and temperature decrease and a new method - dense material cooling under pressure (DMCUP). The latter method allowed the synthesis of dense samples with diameters up to $20 \mathrm{~mm}$ and thicknesses up to $10 \mathrm{~mm}$ from commercial $\mathrm{MgB}_{2}$ powder. Optimal conditions for the DMCUP method with glass encapsulation (maximum pressure $200 \mathrm{MPa}$, maximum temperature $1000{ }^{\circ} \mathrm{C}$ over $200 \mathrm{~min}$, and cooling under pressure) resulted in a dense material with a sharp superconducting transition at $38.5 \mathrm{~K}$. This method employs a pressure which is one order of magnitude less than previously reported for pressure assisted sintering of dense material and can be scaled to larger sample sizes and complex shapes. The data for density, microhardness, fracture toughness and sound speed as well as superconducting properties for bulk magnesium diboride are presented. Ball milling the powder enhances sintering and results in a more homogeneous final microstructure.
\end{abstract}

PACS: 74.62Bf; 74.70.-b

Keywords: $\mathrm{MgB}_{2}$ bulk, HIPing, cooling under pressure, ball milling, density, microhardness, fracture toughness, sound speed, superconducting properties.

*Corresponding author. Nesterenko V.F. Tel.: (858) 822-0289; fax: (858) 534-5698. E-mail address: vnesterenko@ucsd.edu

\section{Introduction}

A relatively high temperature superconducting transition was recently found in the non-oxide compound $\mathrm{MgB}_{2}[1,2]$. Due to the particular properties of this material such as brittleness and 
decomposition at relatively low temperatures, about $1000^{\circ} \mathrm{C}$, fully dense solid samples required for many applications and for fundamental investigations of the superconducting properties are difficult to synthesize.

Superconductivity in $\mathrm{MgB}_{2}$ was discovered in material prepared by Nagamatsu et al. from a mixture of $\mathrm{Mg}$ and B powder [1]. Pressed pellets were hot isostatically pressed at $700{ }^{\circ} \mathrm{C}$ in an argon atmosphere at a pressure of $196 \mathrm{MPa}$ for 10 hours. No encapsulation step was reported. The behavior of the magnetic susceptibility of the sample below the superconducting transition is similar to that of the commercially available $\mathrm{MgB}_{2}$ powder [2,3,4]. Using $\mathrm{MgB}_{2}$ powder (Alfa Aesar, Inc) as a starting material, Jung et al. [2,3] and Takano et al. [4] employed a 12-mm cubic multi-anvil-type press at a pressure of $3000 \mathrm{MPa}$ and a temperature of $950{ }^{\circ} \mathrm{C}$ to synthesize samples with diameters of $\sim 4.5 \mathrm{~mm}$ and heights of $\sim 3.3 \mathrm{~mm}$. It is not clear why such a high level of pressure was required to synthesize a fully dense sample and the natural limitations of a multi anvil press preclude the use of this method for applications requiring samples of large size and complex shape. Therefore it is important to develop a method which will allow scaling of the sample sizes and synthesis of complex shapes.

The main goal of our research is the experimental investigation of the possibility of forming dense samples of $\mathrm{MgB}_{2}$ with high quality superconducting properties under high pressure, which will also make the synthesis of large scale samples of complex shape possible. An additional objective is to investigate the effect of high energy ball milling (SPEX 8000) of the initial powder on the microstructure of the synthesized material and the influence it has on mechanical and superconducting properties.

It was demonstrated for other metallic and ceramic materials that high energy plastic deformation (mechanical milling, dynamic shock densification) combined with traditional sintering 
or HIPing can increase sinterability of powder, reduce final grain size, and improve mechanical properties. One of the reasons for this behavior is the increase of the driving force for the nucleation and the growth of new grains due to the defect structure created during high energy plastic deformation. Additional reasons can be connected with the increased diffusion transport caused by the mechanically induced defect structure and reduction of particle size, and with the enhanced contribution of grain boundary sliding during viscoplastic flow $[5,6,7,8,9]$. No results are published on the structural modification and superconducting properties of $\mathrm{MgB}_{2}$ caused by high energy treatment in combination with sintering or HIPing.

\section{Experiment}

Magnesium diboride powders of -325 mesh size and $98 \%$ purity were obtained from Alfa Aesar, Inc. One batch of powder was milled in a SPEX 8000 ball mill with WC balls and a WC lined vessel for approximately 30 minutes with a balls to powder ratio of approximately 7:1 (mass of powder $5 \mathrm{~g}$ ). Both milled and non-milled $\mathrm{MgB}_{2}$ powders were separately placed in their own closed cylinders made from tantalum foil. Each cylinder was covered with a zirconium foil which served as a getter. The whole assembly was sealed in a pyrex capsule under a vacuum of $10^{-2}$ torr.

HIPing was carried out in an ABB Mini-HIPer using two different cycles: a "standard" HIPing cycle II [9] and the DMCUP method [10,11]. In the first method, the maximum temperature was $1000{ }^{\circ} \mathrm{C}$ and the pressure was $200 \mathrm{MPa}$ with a subsequent simultaneous decrease of pressure and temperature (Fig. 1). The "standard" HIPing of mechanically milled powders in these conditions resulted in solid material with a gold color, but a macrocrack appeared in the middle part of the sample despite its relatively small size of about $5 \mathrm{~mm}$ diameter (Figure 2). HIPing of the nonmilled commercial powder in the same cycle did not result in a solid compact although the material 
was densified (Fig. 3). We speculate that this dramatic difference is due to the enhanced diffusion caused by the defect structure of the ball milled powder and the reduction of the particle size. Further research is under way to explain this activation of sintering due to the high energy treatment of the $\mathrm{MgB}_{2}$ powder.

The DMCUP HIPing procedure was applied under two conditions. In the first schedule the maximum temperature was $850{ }^{\circ} \mathrm{C}$ and the pressure was $200 \mathrm{MPa}$. The resulting samples were dense (density $2.39 \mathrm{~g} / \mathrm{cm}^{3}$ for milled and $2.49 \mathrm{~g} / \mathrm{cm}^{3}$ for non-milled powder, experimental error $3 \%$ ), but contained local pores, and the superconducting transition based on magnetic susceptibility measurements was similar to that obtained for the initial powder.

The second condition of the DMCUP procedure, which resulted in the best mechanical and superconducting properties, used an increased temperature of $1000^{\circ} \mathrm{C}$ with the same pressure of $200 \mathrm{MPa}$. A corresponding HIPing cycle for this schedule is presented in Figure 4. The microstructures of the sintered samples are presented in Figure 5. We can clearly see that ball milling of the powder results in a more homogeneous microstructure similar to that produced by the "standard" HIPing cycle but without macrocracking in the central part of the sample. Small cracks were present in the periphery of the sample and were caused by sharp corners on the Ta container wall.

The critical feature of this method which employs dense material cooling under pressure (DMCUP) $[10,11]$ is the delay of the pressure decrease with respect to temperature (Figure 4). The temperature history in standard HIPing cycle and in DMCUP method is very similar (Figs. 1 and 4). We interpret the completely different results produced by the "standard" HIPing cycle and the DMCUP method for the "as is" and milled powders as the influence of the pressure in the latter 
method during the cooling stage which helps to prevent cracking under thermal gradients and effectively prolong pressure assisted sintering on the stage of cooling.

Despite the clear advantage of the DMCUP method in comparison to the "standard" HIPing cycle with a similar temperature cycle we do not consider the selected parameters for both approaches to be fully optimized. It is possible that a slow and coordinated decrease of temperature and pressure in the "standard" HIPing cycle could result in crack free and fully dense solid samples. Based on our experiments only a slight increase in the temperature hold of "as is" material can result in its sintering in the "standard" HIP cycle.

\section{Results and discussion}

\subsection{Mechanical properties}

Because the "standard" HIP cycle II at investigated conditions did not result in crack free solid samples we will only characterize materials obtained with the DMCUP method. The density of the synthesized material using the optimized DMCUP method was $2.666 \mathrm{~g} / \mathrm{cm}^{3}$ (experimental error $\left.\pm 0.004 \mathrm{~g} / \mathrm{cm}^{3}\right)$ and appeared to be higher than the reported theoretical density based on X-ray measurements $\left(2.625 \mathrm{~g} / \mathrm{cm}^{3}\right)$ [12] and similar to the density reported in [4] after pessure assisted sintering at $3000 \mathrm{MPa}$. Measurements of density were made based on an ASTM B 328 standard by weighing samples immersed in water. The experimental error was found based on the comparison

of the density measurements of pure aluminum samples (wire, 99.999\% $\mathrm{Al}$ ) with the known density of solid aluminum, $2.6989 \mathrm{~g} / \mathrm{cm}^{3}$ [13]. This comparison was made with the mass of the $\mathrm{Al}$ sample close to the mass of the $\mathrm{MgB}_{2}$ sample; these two materials have very similar densities.

The DMCUP method allows us to sinter relatively large dense samples suitable for the sound velocity measurements. A sample with thickness $2.8 \mathrm{~mm}$ and diameter $16 \mathrm{~mm}$ and parallel sides 
was machined for this purpose. The sound speed was measured with a Panametrics pulser-reciever Model 5072 PR using a transducer of $0.125 "$ diameter and $20 \mathrm{MHz}$ frequency. The signals were detected using an oscilloscope (Tectronix Inc.). The instrument was calibrated using a standard 1018 steel test block supplied. The longitudinal sound speed for synthesized $\mathrm{MgB}_{2}$ was found to be $6340 \mathrm{~m} / \mathrm{s}$.

The high density and strength of the synthesized material makes it possible to prepare a polished shiny surface of mirror quality suitable for microhardness measurements. It is important that the indentations are acceptable for the measurements of Vickers microhardness in brittle materials [14]. The average Vickers microhardness of the samples measured under a load of $1 \mathrm{kgf}$ was equal to $10.4 \mathrm{GPa}$ (diagonal length $2 \mathrm{a}=41.8 \mu \mathrm{m}$ ) for the sample prepared from non-milled powder and 11.7 GPa (diagonal length $2 \mathrm{a}=39.4 \mu \mathrm{m}$ ) for the sample from milled powder.

As shown in Fig. 6, during the microhardness measurements transgranular macrocracks (with average lengths of about $96.6 \mu \mathrm{m}$ and $86.3 \mu \mathrm{m}$ calculated from the tip to tip (marked as $2 \mathrm{c}$ ) for non-milled and milled samples respectively) emanated from all four corners of the indentation, which is a typical feature for brittle materials. The presented indentation's shape is typical for fully dense ceramic materials. These types of cracks satisfying the condition $\mathrm{c} \geq 2 \mathrm{a}$ can be used to estimate the fracture toughness using the value of Young's modulus and based on the semiemperical equation [15] $K_{\mathrm{C}}=0.0226(E P)^{1 / 2} a c^{-3 / 2}$, where $E$ is Young's modulus, $P$ is the load used to produce the indentation $(9.807 \mathrm{~N})$, and $a$ and $c$ are the lengths of the diagonal and the crack as shown in Fig. 6. The Young's modulus of the sample (79.6 GPa) was estimated based on measured longitudinal sound speed and density, taking Poisson's ratio as a first approximation to be equal to 0.3 . 
The calculated fracture toughness $K_{\mathrm{C}}$ was found to be $1.26 \mathrm{MPa} \cdot \mathrm{m}^{1 / 2}$ and $1.40 \mathrm{MPa} \cdot \mathrm{m}^{1 / 2}$ for samples HIPed from non-milled and milled powders, respectively. This value of fracture toughness is between the value for monocrystalline silicon and monocrystalline $\mathrm{Al}_{2} \mathrm{O}_{3}$ (sapphire) [15].

\subsection{Superconducting properties}

DC magnetic susceptibility $\chi_{\mathrm{dc}}$ measurements were made with a commercial Quantum Design SQUID magnetometer in a magnetic field of 10 Oe. First, the sample was cooled in zero magnetic field from above its superconducting transition temperature down to $5 \mathrm{~K}$, and then a magnetic field of 10 Oe was applied. Zero field cooled (ZFC) susceptibility data were thus obtained by measuring the magnetization as the sample was warmed to $60 \mathrm{~K}$. Field cooled (FC) data were collected from magnetization measurements as the sample was cooled down to $5 \mathrm{~K}$ with the magnetic field still applied. Resistivity measurements were made using a standard four-probe technique in a commercial Quantum Design Physical Properties Measurement System (PPMS).

The superconducting characteristics of untreated powder and three HIPed samples are compared in Figure 7 as plots of $\chi_{\mathrm{dc}}$ vs. T where the data were normalized to their values at $5 \mathrm{~K}$ and $60 \mathrm{~K}$. In all of the samples there is a considerable difference between the FC and the ZFC curves, consistent with the pinning expected in a Type II superconductor. However, the initial powder (sample A) and the sample HIPed at $850{ }^{\circ} \mathrm{C}$ (sample B) (see Figure 7) also show a significant magnetic moment in the FC data. In contrast, the two samples HIPed at $1000{ }^{\circ} \mathrm{C}$, samples $\mathrm{C}$ and $\mathrm{D}$ (non-milled and milled, respectively; see Figure 7), exhibit no diamagnetism in their FC curves. The crucial impact of the full density and cohesivness of the samples prepared by the DMCUP 
method at $1000{ }^{\circ} \mathrm{C}$ on the pinning strength can be clearly distinguished in comparison with the sample from the initial powder and the sample HIPed at $850{ }^{\circ} \mathrm{C}$.

Electrical resistivity $\rho$ vs. T data for both of the samples HIPed at $1000^{\circ} \mathrm{C}$ (samples $\mathrm{C}$ and D) are shown in Figure 8 between $7 \mathrm{~K}$ and $300 \mathrm{~K}$. The shapes of the $\rho(\mathrm{T})$ curves for these samples above the superconducting transition are reminiscent of a typical metal. The sample HIPed at 850 ${ }^{\circ} \mathrm{C}$ (sample B) exhibited a prohibitively large resistance at room temperature, and thus was not measured. A residual resistivity ratio (RRR) between 300 and $40 \mathrm{~K}$ for samples from the as is powder was found to be 3.6 which is similar to the RRR $=3$ for samples synthesized from commercial poweder at $3000 \mathrm{MPa}$ [3]. For samples prepared from milled powders this ratio was equal to 2.7 with a higher resistivity at $300 \mathrm{~K}$. This difference may be interpreted as caused by smaller grain sizes in the sample HIPed from milled powder.

The superconducting transitions of the two samples HIPed at $1000{ }^{\circ} \mathrm{C}$ and $200 \mathrm{MPa}$ using the DMCUP method are extremely sharp in both dc magnetic susceptibility and electrical resistivity data (Figures 7 and 8). These measurements are consistent with those obtained on samples prepared at pressures of $30 \mathrm{kbar}$ in a multi-anvil-type press [2,3,4] and improved over those attained for the HIPed samples of $\mathrm{MgB}_{2}$ (probably without encapsulation) synthesized by Nagamatsu et al. [1]. The superconducting properties of the materials synthesized in this work under various treatments are shown in Table 1 . We define the transition temperature $T_{C}$ as the temperature at which the susceptibility or resistivity is half of its final value and the transition width $\Delta \mathrm{T}_{\mathrm{C}}$ as the difference between the temperatures at $10 \%$ and $90 \%$ of the final value. Further investigation needs to be done to determine whether the difference in $T_{C}$ and $\Delta T_{C}$ between the samples HIPed at $1000{ }^{\circ} \mathrm{C}$ is due to the ball milling or is simply within experimental error. 
Additional data on the superconducting properties of samples prepared from non-milled powder are presented in a separate paper [16].

\section{Conclusions}

In summary, dense, bulk, crack free materials exhibiting sharp superconducting transitions were synthesized using the dense material cooling under pressure (DMCUP) method based on hot isostatic pressing, which can be utilized at significantly lower pressures (200 MPa) than previously reported. The superconducting properties are similar to those of samples synthesized at $3000 \mathrm{MPa}$ using a multi anvil press. In comparison with the latter method, our method allows scaling of the size of the samples and the manufacture of complex shapes. The mechanical properties (density $2.666 \mathrm{~g} / \mathrm{cc}$, sound speed $6340 \mathrm{~m} / \mathrm{s}$, microhardness 10.4 and $11.7 \mathrm{GPa}$, fracture toughness 1.26 and 1.40 $\mathrm{MPa} \cdot \mathrm{m}^{1 / 2}$ for samples sintered from non-milled and milled powders, respectively) were experimentally measured.

\section{Acknowledgements}

This research was supported by the U.S. Department of Energy under Grant No. DE-FG0386ER-45230.

\section{References}


[1] J. Nagamatsu, N. Nakagava, T. Muranaka, Y. Zenitani, and J. Akimitsu, Nature 410 (2001) 63.

[2] C.U. Jung, M-S. Park, W.N. Kang, M-S. Kim, S.Y. Lee, and S-I. Lee, cond-mat/0102215 (2001).

[3] C.U. Jung, M-S. Park, W.N. Kang, M-S. Kim, S.Y. Lee, and S-I. Lee, Physica C, 353 (2001) 162.

[4] Y. Takano, H. Takeya, H. Fujii, H. Kumakura, T. Hatano, K. Togano, H. Kito, and H. Ihara, Applied Physics Letters, 78 (2001) 2914.

[5] R.A. Graham, Solids Under High-Pressure Shock Compression, Springer-Verlag, New York, 1993.

[6] A.V. Molotkov, A.B. Notkin, D.V. Elagin, V.F. Nesterenko, and A.N. Lazaridi, Combustion, Explosion, and Shock Waves, 27 (1991) 377.

[7] V.F. Nesterenko, V.E. Panin, S.N. Kulkov, , and A.G. Melnikov, High Pressure Research, 10 (1992) 791.

[8] M.A. Morris and M. Leboeuf, Materials Science and Engineering, A224 (1997) 1.

[9] W.B. Eisen, D.C. Hebeisen, Principles and Process Modeling of Higher-Density Consolidation. In: Powder Metal Technologies and Applications, Vol. 7, ASM Handbook, ASM International, Materials Park, Ohio, 1998, pp. 590 604.

[10] S.S. Indrakanti, V.F. Nesterenko, M.B. Maple, N.A. Frederick, W.M. Yuhasz, Shi Li, and E.J. Freeman. Hot Isostatic Pressing of Bulk Magnesium Diboride and Superconducting Properties. Presentation at session “Superconductivity in $\mathrm{MgB}_{2}, " 2001$ MRS Spring Meeting, San Francisco, April 17, 2001.

[11] S.S. Indrakanti, V.F. Nesterenko, M.B. Maple, N.A. Frederick, W.M. Yuhasz, Shi Li, and E.J. Freeman, condmat/0105485 (2001).

[12] 1996 JCPDS - International Center for Diffraction Data, card number 38-1369.

[13] CRC handbook of chemistry and physics: a ready-reference book of chemical and phyical data. Editor-in-chief, David R. Lide. 75th ed. (1994-95 ed.) Boca Raton, FL. CRC Press, p. 4-3.

[14] G.D. Quinn, Indentation Hardness Testing of Ceramics. In: Mechanical Testing and Evaluation, Vol. 8, ASM Handbook, ASM International, Materials Park, Ohio, 2000, pp. 244 - 251.

[15] G.R. Anstis, P. Chantikul, B.R. Lawn, and D.B. Marshall, Journal of the American Ceramic Society, 64 (1981) 533.

[16] N.A. Frederick, Shi Li, M.B. Maple, V.F. Nesterenko, and S.S. Indrakanti, Physica C (2001) submitted. 
Table 1

Superconducting transition characteristics determined from dc magnetic susceptibility $\chi_{\mathrm{dc}}$ and electrical resistivity $\rho$ measurements for Alfa Aesar $\mathrm{MgB}_{2}$ powder under various treatments. $\mathrm{T}_{\mathrm{C}}$ is defined as the temperature at which the susceptibility or resistivity is $50 \%$ of its final value. $\Delta \mathrm{T}_{\mathrm{C}}$, the width of the superconducting transition, is defined as the difference between the temperatures at $10 \%$ and $90 \%$ of the final value.

\begin{tabular}{|c|l|c|c|c|c|}
\hline \multicolumn{2}{|c|}{ Alfa Aesar MgB powder } & \multicolumn{2}{c|}{$\chi_{\mathrm{dc}}$} & \multicolumn{2}{c|}{$\rho$} \\
\hline Sample & \multicolumn{1}{|c|}{ Treatment } & $\mathrm{T}_{\mathrm{C}}(\mathrm{K})$ & $\Delta \mathrm{T}_{\mathrm{C}}(\mathrm{K})$ & $\mathrm{T}_{\mathrm{C}}(\mathrm{K})$ & $\Delta \mathrm{T}_{\mathrm{C}}(\mathrm{K})$ \\
\hline $\mathrm{A}$ & As received & 36.79 & 12.54 & - & - \\
\hline $\mathrm{B}$ & HIPed @ $850{ }^{\circ} \mathrm{C}$ & 36.75 & 10.57 & - & - \\
\hline $\mathrm{C}$ & Non-milled, HIPed @ $1000^{\circ} \mathrm{C}$ & 37.66 & 0.75 & 38.50 & 0.85 \\
\hline $\mathrm{D}$ & Milled, HIPed @ $1000^{\circ} \mathrm{C}$ & 37.24 & 0.35 & 38.11 & 0.76 \\
\hline
\end{tabular}




\section{Figure captions}

Figure 1. Temperature and pressure histories of the "standard" hot isostatic pressing cycle II.

Figure 2. Cracking of samples sintered from ball milled powder in the conditions of the "standard" HIPing cycle II.

Figure 3. Nonsintered sample from commercial powder after the "standard" HIPing cycle II, under the same conditions as for the HIPing of the ball milled powder.

Figure 4. Temperature and pressure histories of the hot isostatic pressing cycle for the DMCUP method.

Figure 5. Microstructures of the "as is" (a) and milled (b) powders after DMCUP method.

Figure 6. Indentations after measurements of Vickers microhardness of "as is" (a) and milled powders (b). Note cracks emanating from the indentation corners.

Figure 7. DC magnetic susceptibility $\chi_{\mathrm{dc}}$ (normalized to values at $5 \mathrm{~K}$ and $60 \mathrm{~K}$ ) versus temperature of Alfa Aesar $\mathrm{MgB}_{2}$ samples. Shown are measurements on the original powder and on samples HIPed using the DMCUP method. 
Figure 8. Electrical resistivity $\rho$ vs. T of Alfa Aesar $\mathrm{MgB}_{2}$ HIPed at $1000{ }^{\circ} \mathrm{C}$ using the DMCUP method. 
HIPing, Cycle II

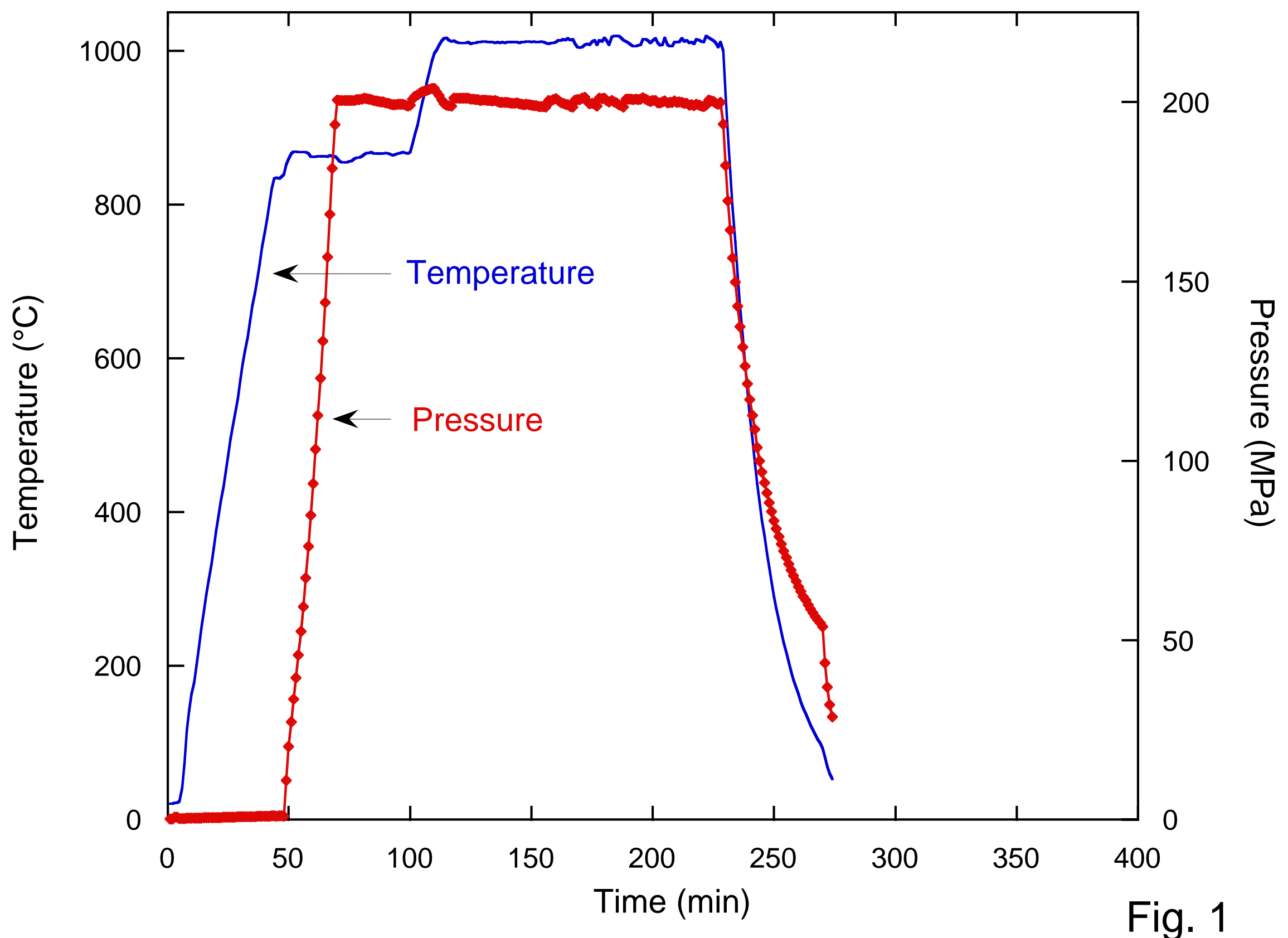




\section{Fig. 2}

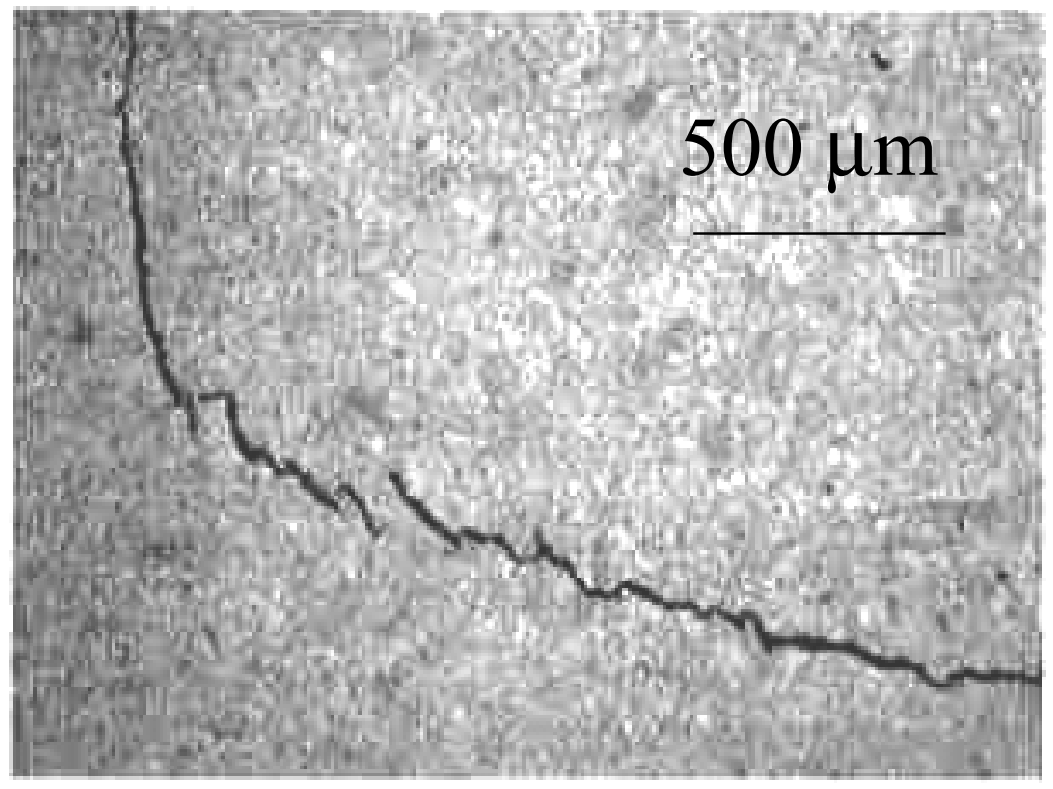

Fig. 3

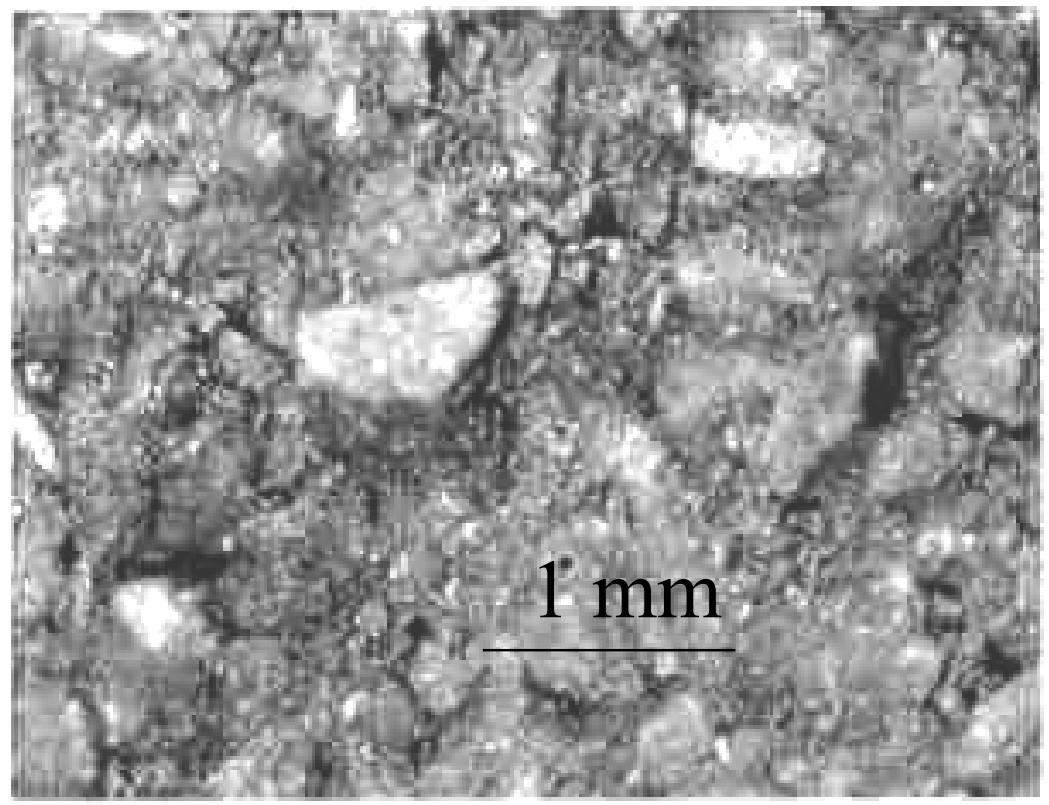


HIPing, DMCUP

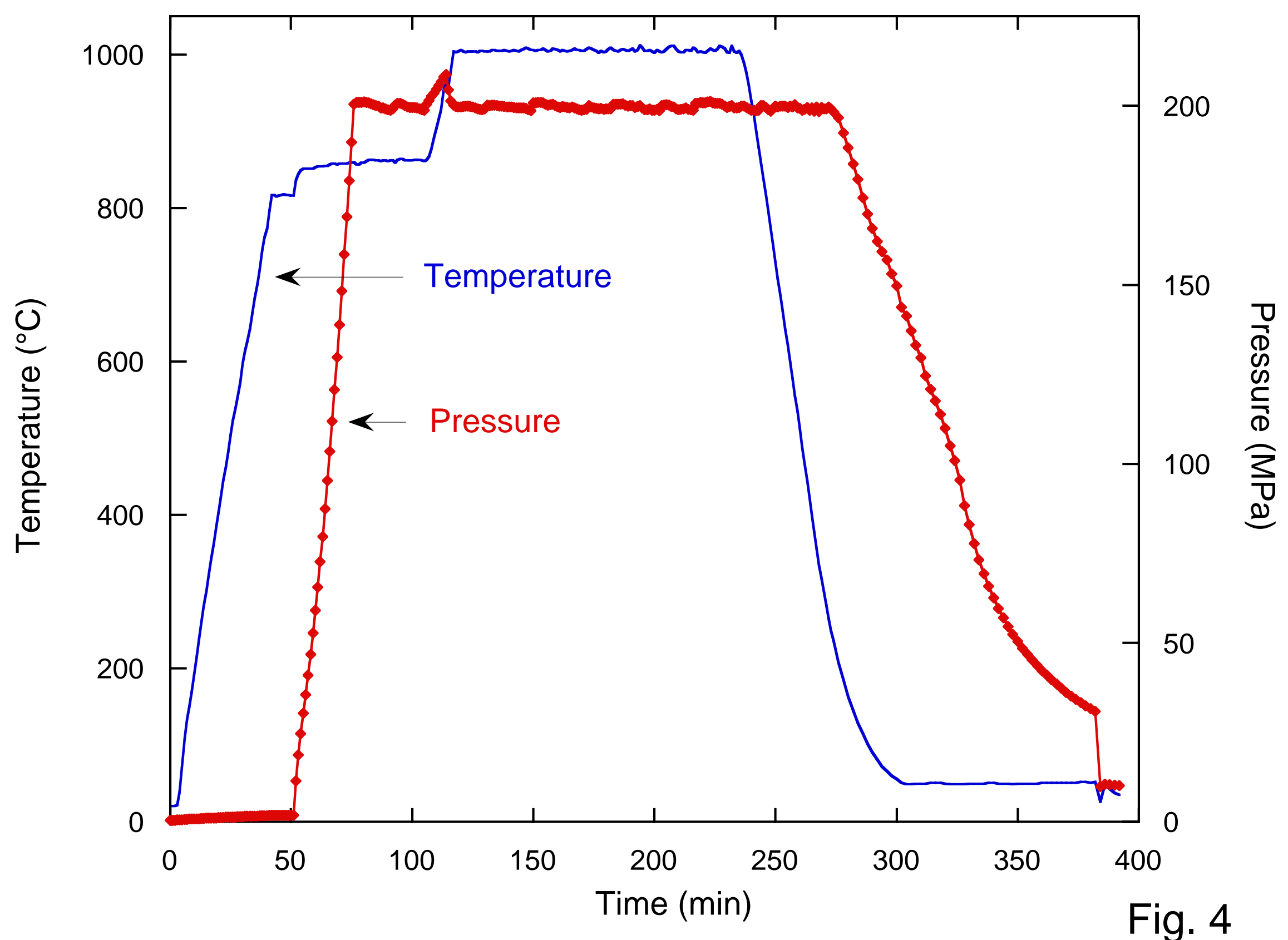




\section{Fig. $5 \mathrm{a}$}

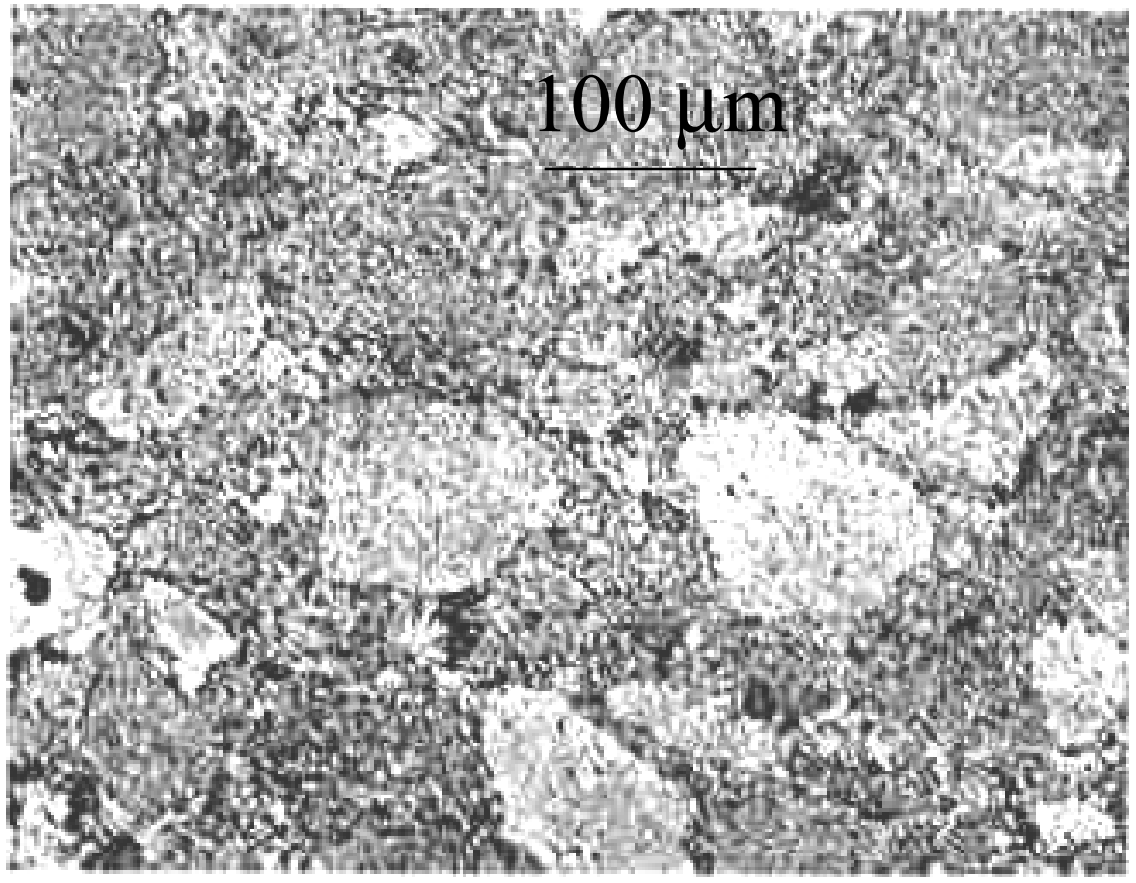

\section{Fig. 5b}

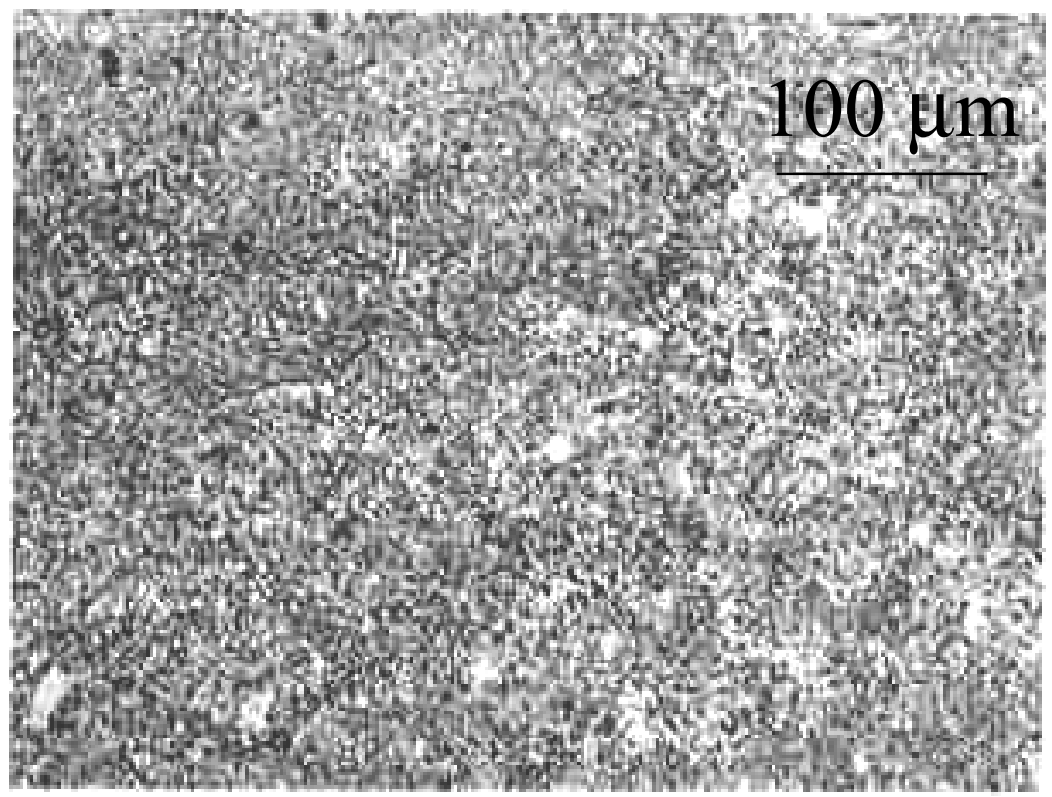




\section{Fig. 6a}

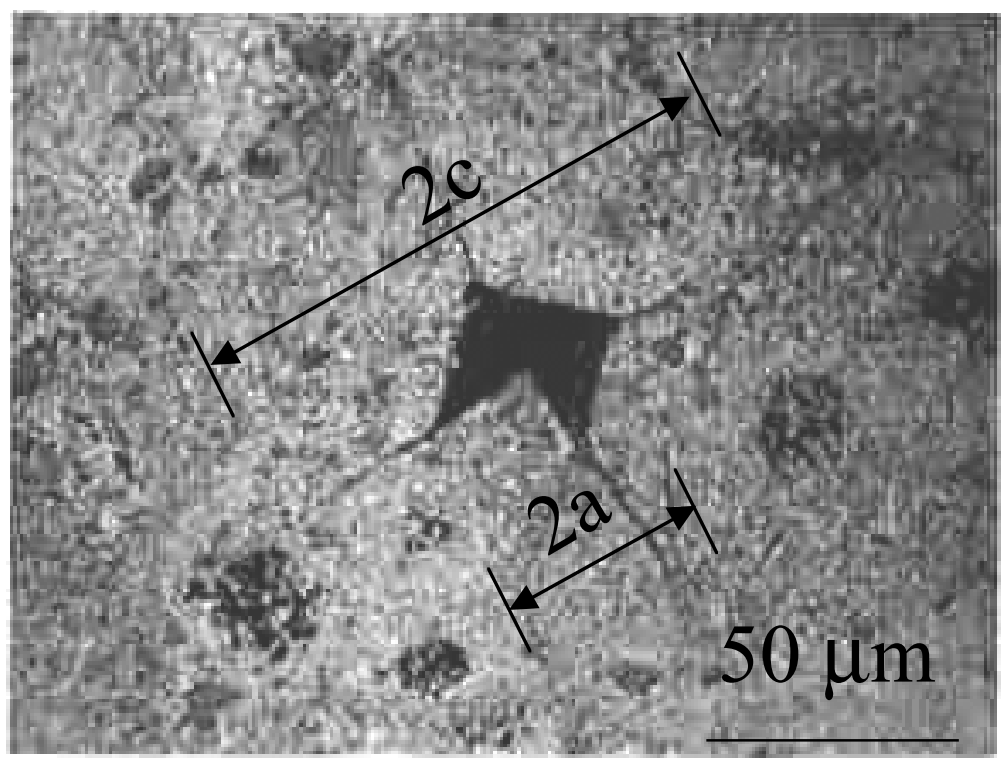

Fig. 6b

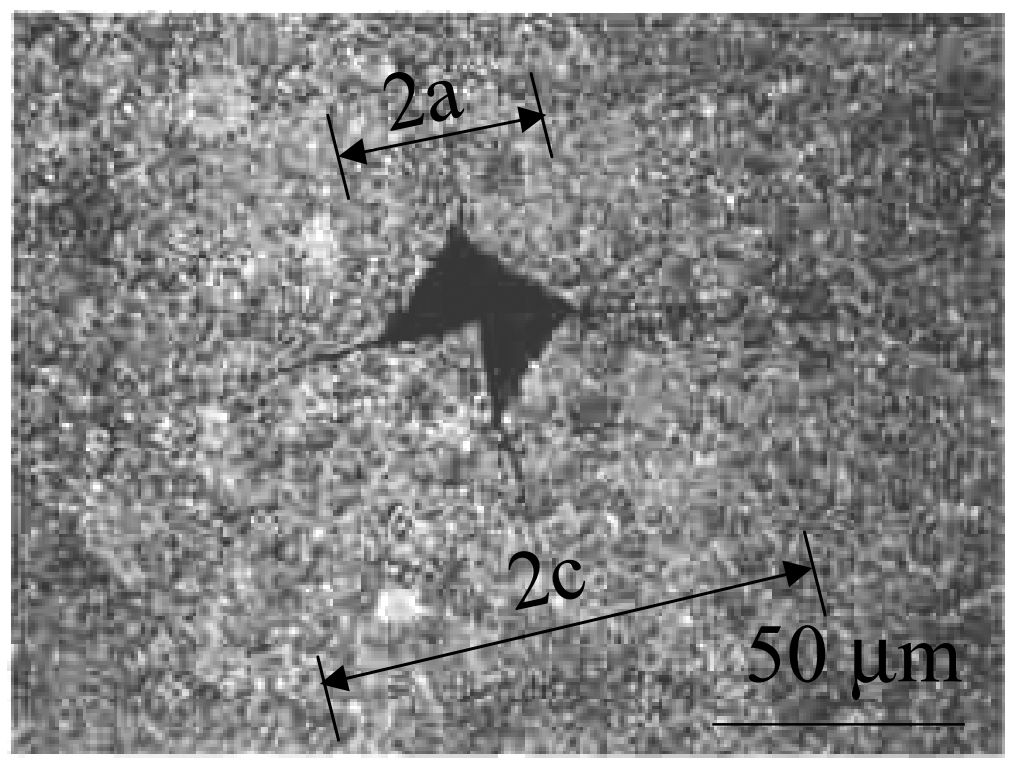




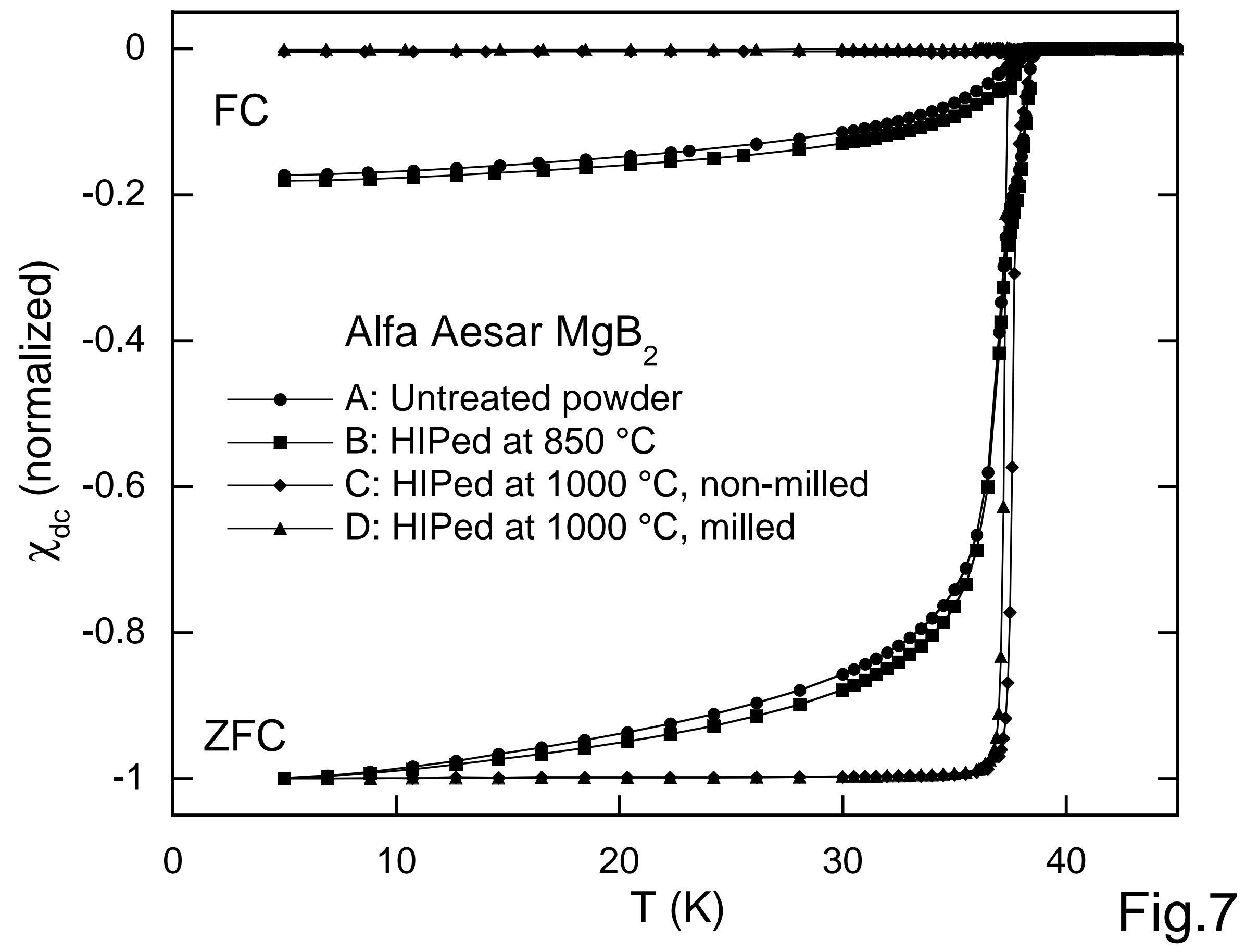




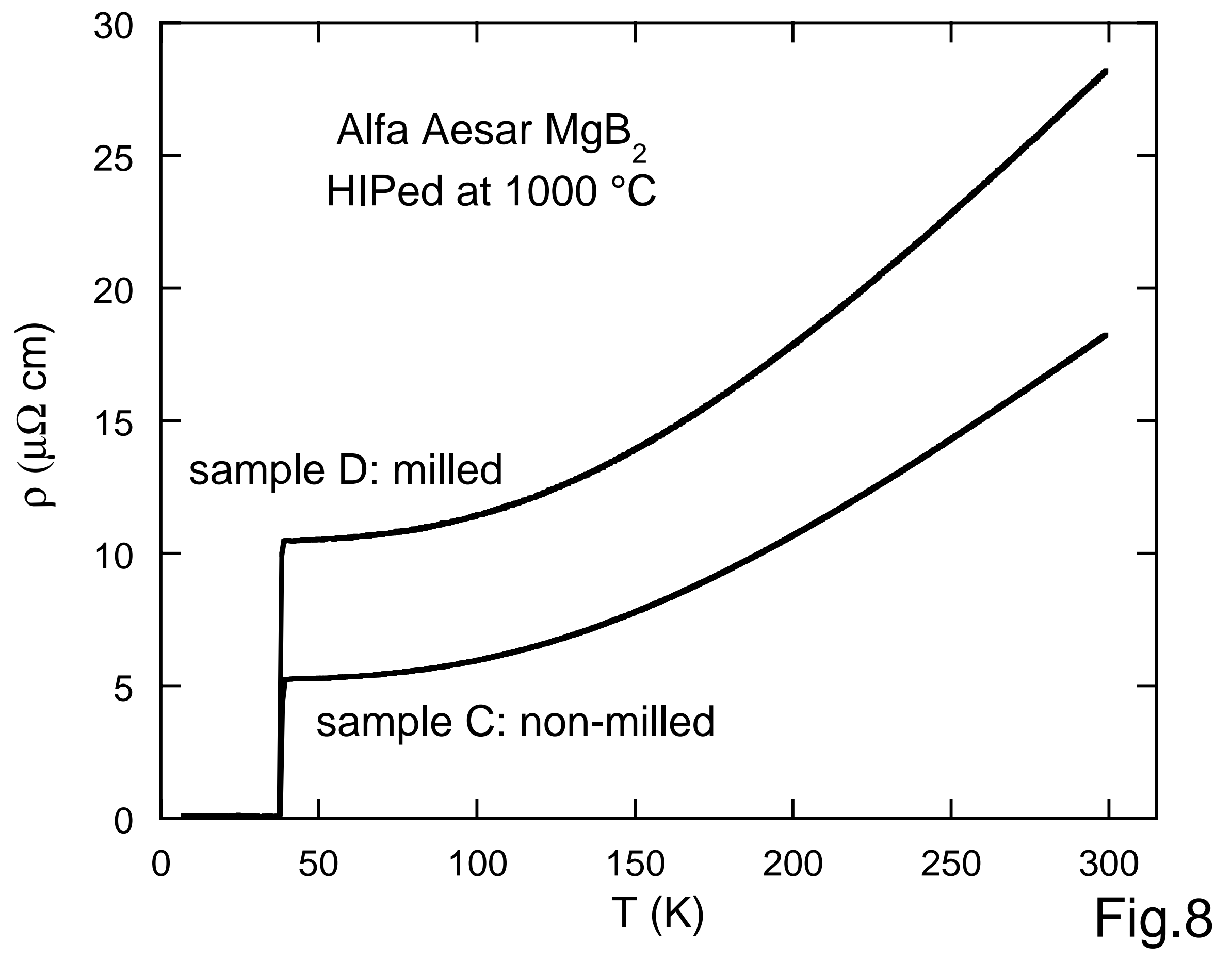

